\title{
Buffering against maladaptive perfectionism in bipolar disorder: the role of self-compassion
}

Short title: Perfectionism and self-compassion in BD

Kathryn Fletcher ${ }^{1 *}$, Yan Yang ${ }^{1}$, Sheri L. Johnson ${ }^{2}$, Michael Berk ${ }^{3,4,5,6,7}$, Tania Perich ${ }^{8,9}$, Sue Cotton ${ }^{5,6}$, Steven Jones ${ }^{10}$, Sara Lapsley ${ }^{11}$, Erin Michalak ${ }^{11}$, Greg Murray ${ }^{1}$.

* Corresponding author: Dr Kathryn Fletcher, Centre for Mental Health, Swinburne University of Technology, PO Box 218, Hawthorn, Victoria, AUSTRALIA. Email: kfletcher@swin.edu.au

Affiliations:

${ }^{1}$ Centre for Mental Health, Swinburne University, Melbourne, Australia

${ }^{2}$ University of California, Berkeley, CA, USA

${ }^{3}$ IMPACT Strategic Research Centre, School of Medicine, Deakin University, Barwon Health, Geelong, Australia

${ }^{4}$ Department of Psychiatry, University of Melbourne, Melbourne, Australia

${ }^{5}$ Orygen, the National Centre of Excellence in Youth Mental Health, Parkville, Australia

${ }^{6}$ Centre for Youth Mental Health, University of Melbourne, Melbourne, Australia

${ }^{7}$ Florey Institute for Neuroscience and Mental Health, Melbourne, Australia

${ }^{8}$ Western Sydney University, Sydney, Australia

${ }^{9}$ School of Psychiatry, University of New South Wales, Sydney, Australia

${ }^{10}$ Spectrum Centre for Mental Health Research, Faculty of Health and Medicine, Lancaster

University, Lancaster, UK

${ }^{11}$ Department of Psychiatry, University of British Columbia, Vancouver, Canada 


\section{Abstract}

Background: Maladaptive perfectionism is a transdiagnostic risk and maintaining factor for a range of mental health conditions, including bipolar disorder (BD). Self-compassion represents a potential protective factor against maladaptive perfectionism, however no studies to date have examined the relationship of these constructs in $\mathrm{BD}$. The aim of the present study was to examine associations between maladaptive perfectionism, self-compassion and symptoms among individuals with BD.

Methods: Baseline data were collected from 302 participants with a DSM-IV diagnosis of BD participating in an international randomised controlled trial. Participants completed measures of maladaptive perfectionism, self-compassion, symptom severity and emotion regulation difficulties. Clinician-administered measures of depression and mania severity were additionally collected. Correlation and mediation analyses were conducted.

Results: Maladaptive perfectionism was positively associated with depression, anxiety and emotion regulation difficulties. Lower levels of self-compassion correlated with greater self-reported depression, anxiety and emotion regulation difficulties. Self-compassion partially mediated relationships between maladaptive perfectionism, depression, anxiety and emotion regulation difficulties.

Limitations: The cross-sectional design limits conclusions about causal relationships between study variables. Results may not be generalizable to other BD populations. The role of maladaptive perfectionism and self-compassion in elevated mood states of BD remains unclear.

Conclusion: Self-compassion represents one mechanism through which maladaptive perfectionism influences symptoms of depression, anxiety and emotion regulation difficulties in BD. Self-compassion represents a modifiable treatment target; individuals with $\mathrm{BD}$ exhibiting maladaptive perfectionistic tendencies may benefit from interventions fostering self-compassion.

Key words: Bipolar disorder, maladaptive perfectionism, self-compassion, mediator, treatment 


\section{Introduction}

Perfectionism is defined as the tendency to set excessively high standards for oneself and to engage in overly critical self-evaluations (Frost et al., 1990). The construct is multidimensional, broadly organised into two superordinate factors: perfectionistic strivings and perfectionistic concerns (Stoeber and Otto, 2006). The former captures a self-oriented striving for perfection combined with high personal standards (conceptually mapping on to adaptive aspects of perfectionism). The latter captures maladaptive perfectionism, characterised by self-criticism, fear of failure and negative evaluation by self or others, concerns over making mistakes and doubts about actions and feelings of discrepancy between one's high standards and actual performance (Stoeber and Otto, 2006). High levels of distress arise from striving to meet unrealistic standards and unattainable goals, driven by a fear of failure and criticism and attempts to escape feelings of inferiority (Lo and Abbott, 2013). Maladaptive perfectionism, the focus of the present study, is a transdiagnostic risk and maintaining factor across a range of mental health conditions including eating disorders, depression and anxiety (Egan et al., 2011), and has been associated with suicidal ideation and behaviour (Hewitt et al., 2006). Controlled studies have demonstrated that treatment of perfectionism results in a reduction of symptoms across clinical disorders (Glover et al., 2007; Riley et al., 2007; Steele and Wade, 2008). Understanding risk and protective factors that influence maladaptive perfectionism is therefore a clinical research priority.

\subsection{Perfectionism in bipolar disorder}

Perfectionistic cognitive styles have been implicated in several psychological models of risk and outcome for bipolar disorder (BD). The transdiagnostic model of mood dysregulation and BD (Mansell et al., 2007) proposes that self-critical or shaming beliefs (constructs linked to perfectionism) are major drivers of maladaptive cognitive appraisals, and that in turn, those maladaptive cognitive appraisals promote the development of depressive and manic symptoms. Similarly, the expanded Behavioural Activation System (Becerra et al., 2013) dysregulation theory of BD (Urošević et al., 2008) describes $B D$ as related to a processing style characterised by perfectionistic standards, with a predominant focus on performance and high levels of self-criticism. Perfectionistic standards are associated with excessive goal striving, similarly associated with risk and outcome in BD (Johnson, 2005; Johnson et al., 2012).

A number of studies indicate that perfectionism is elevated in $B D$, and more importantly, that it is predictive of mood swings among those with BD. Cross-sectionally, for example, relative to controls, perfectionistic dysfunctional attitudes (Scott et al., 2000), BAS-relevant cognitive styles (Fletcher et al., 2013) and significantly higher perfectionism scores have been observed in BD (Jones et al., 2005). 
High Dysfunctional Attitude Scale scores reflecting maladaptive perfectionism have been observed among those with BD after controlling for symptoms (Alloy et al., 2009; Lam et al., 2004), and among those with remitted BD as compared to controls (Wright et al., 2005). Longitudinally, perfectionism predicted the onset of manic and hypomanic episodes in one study (Alloy et al., 2009), while in another study, self-criticism, performance focus and high self-standards interacted with congruent life events to predict depressive and manic symptoms in those with bipolar II disorder and cyclothymia (FrancisRaniere et al., 2006). After controlling for baseline depressive and manic symptoms, perfectionistic cognitive styles mediated the association between bipolar spectrum disorders and anxiety comorbidity and increased depressive symptoms at one-year follow-up (O'Garro-Moore et al., 2015).

There appears to be some specificity in the nature of perfectionism observed among those with BD. Items concerning performance and achievement standards appear more sensitive to the disorder than do those regarding dependency and the need for approval (Lam et al., 2004). More recently, differing dimensions of perfectionism (e.g., self-oriented perfectionism versus socially-prescribed perfectionism) have been found to be uniquely positively associated with chronic depressive and manic symptoms in BD pedigrees, mediating the relationship between anxiety/stress and depressive and manic symptoms (Corry et al., 2017). Taken together, empirical findings to date suggest perfectionism may play an important role in BD symptoms and outcomes.

\subsection{Self-compassion: an antidote to perfectionism?}

Self-compassion, one way of relating to oneself in a kind and healthy manner, is garnering increasing interest in psychological research and treatment. Three components of self-compassion have been described (Neff, 2003): (i) self-kindness (as opposed to self-criticism), (ii) common humanity (rather than separating and isolating oneself) and (iii) mindfulness (as opposed to over-identifying with one's painful experiences). A self-compassionate stance acknowledges the suffering and pain that is experienced by the self, in the context of the human condition. By fostering self-kindness and selfacceptance through actions such as meditation, writing, or challenging negative self-talk, a selfcompassionate approach may theoretically buffer against the harsh and self-critical style associated with maladaptive perfectionism (Lowens, 2010). Empirical data from non-clinical populations is supportive of this idea. Self-compassion partially mediated the association between perfectionism and depression in university students (Mehr and Adams, 2016); while in another study (Ferrari et al., 2018), moderation analyses revealed that self-compassion was associated with diminished strength of the relationship between maladaptive perfectionism and depression in two independent samples (adolescents and adults). In a related study, we found that self-compassion mediated the relationship between vulnerability to BD and psychological distress in a non-clinical sample (Yang et al, 2018). In 
one cross-sectional study, those with remitted BD reported significantly lower mean levels of selfcompassion relative to healthy controls (Døssing et al., 2015). On the subscales of the measure used (the Self-Compassion Scale)(Neff, 2003), those with BD reported significantly lower scores on SelfKindness and significantly higher scores on Self-Judgment (Døssing et al., 2015).

Self-compassion is a skill that can be cultivated as a practice, requiring the individual to be mindfully aware of thoughts and feelings they experience, without judgment (Neff, 2003). As such, selfcompassion may represent a modifiable treatment target, with growing evidence supporting the efficacy of self-compassion-focused therapies for a range of conditions including borderline personality disorder (Soler et al., 2015), social anxiety disorder (Koszycki et al., 2016), major depressive disorder (MDD) (Krieger et al., 2013) and psychosis (Braehler et al., 2013). A systematic review on the benefits of compassion-focused therapy (CFT) indicated this approach holds promise for mood disorders (including BD) - particularly those high in self-criticism (Leaviss and Uttley, 2015).

\subsection{Aims of the study}

The aim of the present study is to examine the relationships between maladaptive perfectionism, selfcompassion and symptoms among individuals with BD. We hypothesise that (i) maladaptive perfectionism will be positively associated with BD symptoms (depression, mania, anxiety, emotion regulation difficulties) and negatively associated with self-compassion, (ii) lower levels of selfcompassion will be related to greater endorsement of bipolar symptoms, and (iii) self-compassion will mediate the relationship between maladaptive perfectionism and BD symptoms.

\section{Materials and methods}

\subsection{Design}

This study involved secondary data analysis of baseline data collected as part of a larger international randomised controlled trial (the ORBIT RCT; Trial registration NCT03197974) investigating the effectiveness of an online self-management program for individuals with 'late stage' BD (defined as 10 or more mood episodes). Study protocol details are outlined elsewhere (Fletcher et al., 2018).

\subsection{Participants and procedures}

Participants (18-65 years of age) who had received a diagnosis of BD from a medical practitioner provided informed consent following study registration online. Other eligibility criteria included a confirmed DSM-IV diagnosis of BD (I, II or NOS) based on the MINI International Neuropsychiatric Interview (MINI)(Sheehan et al., 1998), no current mood episode (assessed via the MINI), no current 
psychotic features or active suicidality, under the care of a medical practitioner. Structured interviews ( $\mathrm{MINI}$, clinician-administered symptom measures) were conducted via telephone by trained Masterslevel clinical research interviewers, with inter-rater reliability checks conducted every three months. Participants then completed an online battery of questionnaires assessing demographic and clinical data, including self-reported measures of perfectionism, self-compassion, depression, anxiety and emotion regulation difficulties (detailed below). Upon completion of baseline assessments, participants were subsequently randomised to the study trial. Baseline data collected for $n=302$ consecutively randomised participants are reported in the present study. The study was approved by the Swinburne University Human Research Ethics Committee (2016/289) and informed consent was obtained from all participants.

\subsection{Measures}

\subsubsection{Maladaptive Perfectionism}

The Almost Perfect Scale-Revised (APS-R)(Slaney et al., 2001) is a widely used self-report measure of adaptive and maladaptive perfectionism. We used a brief version of the APS-R, the Short revised Almost Perfect Scale (SAPS)(Rice et al., 2014), that has demonstrated adequate internal consistency (up to Cronbach's $\alpha=0.87$ ), convergent and discriminant validity with other indicators of perfectionism and related constructs (e.g., conscientiousness, neuroticism, emotion regulation and depression). The SAPS Discrepancy subscale (SAPS-D), used in the present study, assesses maladaptive perfectionism, including the extent to which the individual perceives their own performance as failing to meet their expected standards. Higher scores indicate higher levels of maladaptive perfectionism.

\subsubsection{Self-compassion}

The 26-item Self-Compassion Scale (SCS)(Neff, 2003) comprises six sub-scales measuring dimensions of global self-compassion: Self-Kindness, Self-Judgement, Common Humanity, Isolation, Mindfulness and Over-Identification. Items are rated on a 5-point Likert scale ( 1 = almost never; 5 = almost always) and can be summed to obtain an overall self-compassion score (higher scores indicating greater selfcompassion). The SCS has sound psychometric properties including good convergent and discriminant validity, high test-retest reliability ( 0.83 over 3 weeks) and internal consistency (Cronbach's $\alpha=.92$ ) (Neff, 2003). A recent examination of the scale's factor structure supports the use of the total score as a reliable measure of self-compassion (Neff et al., 2017) and is therefore reported in the present study.

\subsubsection{Mood symptoms}




\section{Depression}

1. The Quick Inventory of Depressive Symptomatology-Self-Report (QIDS-SR)(Rush et al., 2003) is a self-report measure of depression symptoms with high internal consistency (Cronbach's $\alpha=0.87$ ) that correlates strongly with established clinician-rated scales including the Hamilton Depression Rating Scale ( $r=0.86$ ) (Rush et al., 2003). Higher scores indicate more severe depression.

2. The Montgomery-Asberg Depression Rating Scale (MADRS)(Montgomery and Asberg, 1979) is a widely-used, valid and reliable measure 10-item clinician-administered scale of depressive symptomatology that is sensitive to change. Higher scores indicate more severe depression. While the MADRS contains one item ('apparent sadness') based on visual observation, prior studies (e.g. Hermens et al., 2006; Kobak et al., 2008) have demonstrated that the full MADRS, including this item, can be administered reliably via telephone. In line with these studies, interviewers in the present study were instructed to be attentive to verbal signs (e.g. tone of voice, rhythm, pace of talking etc.) to compensate for this item in assessing level of sadness.

Mania

The Young Mania Rating Scale (YMRS)(Young et al., 1978) is an 11-item clinician-administered scale assessing symptoms of mania. This scale has well-established reliability and validity (Young et al., 1978; Miller et al., 2009). Higher scores indicate more severe mania. Similar to the MADRS, the YMRS contains one item ('appearance') based on visual observation. Interviewers were trained to use probe questions (e.g. have others noticed changes in the way you dress) to account for this item. As noted above and overviewed by Hermens et al. (2006), studies examining comparability of face-to-face and telephone-administered interviews capturing data on psychiatric symptoms indicate the latter are as valid as those obtained by the former.

\section{Anxiety}

The Depression Anxiety Stress Scale-21 items (DASS-21)(Lovibond and Lovibond, 1995) is a valid and reliable self-report measure of symptoms experienced over the previous week. The DASS-Anxiety (DASS-Anx) subscale score was used in the present study, measuring anxiety symptoms including physiological arousal and fear based emotions. High internal consistency (Cronbach's $\alpha=.87$ ) has been reported for the DASS-Anx subscale (Antony et al., 1998). Higher scores indicate more severe anxiety.

\section{Emotion regulation difficulties}

The Difficulties in Emotion Regulation Scale (Gratz and Roemer, 2004) is a widely-used 36-item multidimensional self-report measure of emotion regulation difficulties. Higher scores indicate more 
difficulty in emotion regulation. The short-form version (DERS-16)(Bjureberg et al., 2016) assesses similar constructs (non-acceptance of negative emotions, inability to engage in goal-directed behaviours when distressed, difficulties controlling impulsive behaviours when distressed, limited access to emotion regulation strategies perceived as effective, lack of emotional clarity). In a large transdiagnostic sample of treatment-seeking adults with emotional disorders (including BD), DERS-16 scores showed strong concordance with the original measure (Hallion et al., 2018). The measure has excellent internal consistency (Cronbach's $\alpha=0.92$ ) and good test-retest reliability (Bjureberg et al., 2016). We used the total DERS-16 score in the present study.

\subsection{Statistical analyses}

Statistical analyses were conducted using IBM ${ }^{\circledR}$ SPSS ${ }^{\circledR}$ Statistics Version 24.0. First, mean and standard deviations, and Cronbach's alpha $(\alpha)$ were reported for each of the scales. Second, Pearson Product Moment correlations $(r)$ were calculated to reflect bivariate associations between perfectionism, selfcompassion and BD symptoms (depression, anxiety, emotion regulation difficulties). Second, mediation analyses were undertaken using a bootstrapped multivariate procedure (Preacher and Hayes, 2008), using the PROCESS macro Version 3 (Hayes and Rockwood, 2017) in IBM SPSS Statistics. Results are based on 5000 bootstrapped samples and $95 \%$ confidence intervals. The indirect effect is considered significant if the upper and lower bounds of the confidence intervals do not contain zero (Preacher and Hayes, 2008). This method maximises power using smaller sample sizes and does not require data to adhere to assumptions of normality (Hayes, 2013). Mediation was investigated by testing significance of the indirect effects of the independent variable on the dependent variable (DV) through the mediator (M). Complete mediation is demonstrated when the IV no longer affects the DV after the mediator has been controlled, making path $c^{\prime}$ zero; partial mediation is demonstrated when the path from the IV to the DV is reduced in absolute size but is still different from zero when the mediator is introduced (Baron and Kenny, 1986). In the present study, self-compassion was tested as a mediator in the relationship between maladaptive perfectionism (the IV) and BD symptoms (the DVs, with separate mediation models examining depression, anxiety and emotion regulation difficulties).

\section{Results}

Mean scores, standard deviations, score range in the sample and Cronbach's $(\alpha)$ for all measures are summarised in Table 1. All measures showed adequate variability and internal consistency.

(INSERT TABLE 1 HERE) 


\subsection{Sample characteristics}

Data were obtained from 302 participants (242 BD I, 54 BD II, 6 BD NOS), with a preponderance of females $(70.5 \%)$ and mean age of 44 years $(S D=11.7$, range $20-65$ years). The majority $(96.7 \%)$ indicated they had taken psychotropic medication during the past month. Demographic details are outlined in Table 2.

(INSERT TABLE 2 HERE)

\subsection{Bivariate correlations}

To test for mediation, significant associations should occur between (a) the predictor (maladaptive perfectionism) and the mediator (self-compassion), (b) the mediator and outcome (BD symptoms), and (c) the predictor and outcome (Baron and Kenny, 1986). As shown in Table 3, correlations were all significant (all $p$ 's $<0.05$ ) in predicted directions for the majority of key variables: (a) maladaptive perfectionism (SAPS-D) was strongly but negatively correlated with self-compassion (SCS), (b) SCS was negatively correlated with clinician-rated depression (MADRS), self-reported depression (QIDS-SR), anxiety (DASS-Anx) and emotion regulation difficulties (DERS-16), (c) SAPS-D positively correlated with QIDS-SR, MADRS, DASS-Anx, and DERS-16. The mediator (self-compassion) did not correlate with the YMRS, therefore mediation analyses were not conducted with this DV.

(INSERT TABLE 3 HERE)

To test for the possible confounding influence of demographic variables (IVs) on remaining key measures (DVs: SAPS-D, SC, MADRS, QIDS-SR, DASS-Anx, DERS), a series of multivariate regression analyses were undertaken. Dummy variables were created for categorical variables. The percomparison alpha level was set to 0.001 to minimise Type I error. Significant relationships were found between marital status and SCS $(F(5,296)=3.4, p<0.05)$; employment status and DASS-Anx $(F(8,293)$ $=2.9, \mathrm{p}<0.05)$; bipolar subtype diagnosis and $\operatorname{MADRS}(F(2,299)=3.9, \mathrm{p}<0.05)$. Therefore, marital status, employment status and bipolar subtype diagnosis were entered as covariates in mediation analyses.

\subsection{Mediation analyses}

Separate parallel mediation models were constructed to test the hypothesis that self-compassion would be a significant mediator of maladaptive perfectionism with depression (Model 1, Figure 1), anxiety (Model 2, Figure 2) and emotion regulation difficulties (Model 3, Figure 3). 


\subsubsection{Model 1: Depression}

As depicted in Figure 1, maladaptive perfectionism was a significant predictor of self-compassion (path a), $b=-1.61, \mathrm{SE}=0.14, \mathrm{t}(297)=-11.26, p<0.01$, and self-compassion was a significant predictor of self-reported depression (path $b$ ), $b=-0.04, \mathrm{SE}=0.01, \mathrm{t}(296)=-2.82, p<0.01$. The total effect was significant, whereby those with higher scores on maladaptive perfectionism reported more severe self-reported depression, $b=0.22, \mathrm{SE}=0.04, \mathrm{t}(297)=5.48, p<0.01$. The indirect effect was significant, whereby self-compassion mediated the relationship between maladaptive perfectionism and selfreported depression, $b=0.07, \mathrm{SE}=0.02,95 \% \mathrm{Cl}[0.02,0.12]$. However, the direct effect remained significant with maladaptive perfectionism continuing to predict self-reported depression (path $\left.c^{\prime}\right), b$ $=0.14, t(296)=3.11, p<0.01$, suggesting partial mediation by self-compassion. Approximately $14 \%$ of the variance in self-reported depression severity was accounted for by the predictors $\left(R^{2}=0.139\right)$.

(INSERT FIGURE 1 HERE)

Turning to clinician-rated depression, mediation was not supported: (i) self-compassion did not significantly predict clinician-rated depression (path b), $\mathrm{b}=-0.03, \mathrm{SE}=0.02, \mathrm{t}(296)=-1.16, \mathrm{p}=0.25$, and ii) the indirect effect was non-significant, whereby self-compassion did not mediate the relationship between maladaptive perfectionism and clinician-rated depression, $b=0.05$, SE $=0.04$, $95 \% \mathrm{Cl}[-0.03,0.12]$.

\subsubsection{Model 2: Anxiety}

Self-compassion was a significant predictor of anxiety (path $b$ ), $\mathrm{b}=-0.03, \mathrm{SE}=0.01, \mathrm{t}(296)=-2.92, \mathrm{p}<$ 0.01. The total effect was significant, whereby those with higher scores on maladaptive perfectionism reported more severe anxiety, $b=0.14, \mathrm{SE}=0.03, \mathrm{t}(297)=4.70, \mathrm{p}<0.01$. The indirect effect was significant, indicating self-compassion mediated the relationship between maladaptive perfectionism and anxiety, $b=0.06, \mathrm{SE}=0.02,95 \% \mathrm{Cl}[0.02,0.09]$. However, the direct effect remained significant with maladaptive perfectionism continuing to predict anxiety (path $\left.c^{\prime}\right), b=0.08, \mathrm{SE}=0.03, \mathrm{t}(296)=$ $2.40, p<0.05$, suggesting partial mediation by self-compassion. Approximately $13 \%$ of the variance in anxiety severity was accounted for by the predictors $\left(R^{2}=0.127\right)$.

(INSERT FIGURE 2 HERE)

\subsubsection{Model 3: Emotion regulation difficulties}


As outlined in Figure 3, self-compassion was a significant predictor of emotion regulation difficulties (path b), $\mathrm{b}=-0.50, \mathrm{SE}=0.03, \mathrm{t}(296)=-14.04, \mathrm{p}<0.01$. The total effect was significant, whereby those with higher scores on maladaptive perfectionism reported more emotion regulation difficulties, $b=$ $1.09, \mathrm{SE}=0.11, \mathrm{t}(296)=9.66, \mathrm{p}<0.01$. The indirect effect was significant, indicating self-compassion mediated the relationship between maladaptive perfectionism and emotion regulation difficulties, $b$ $=0.80, \mathrm{SE}=0.10,95 \% \mathrm{Cl}[0.60,1.00]$. However, the direct effect remained significant with maladaptive perfectionism continuing to predict emotion regulation difficulties (path $\left.c^{\prime}\right), b=0.29, \mathrm{SE}=0.10, \mathrm{t}(296)$ $=2.72, p<0.01$, suggesting partial mediation by self-compassion. Approximately $56 \%$ of the variance in emotion regulation difficulties was accounted for by the predictors $\left(R^{2}=0.557\right)$.

\section{(INSERT FIGURE 3 HERE)}

\section{Discussion}

This is the first study to investigate the relationships between maladaptive perfectionism, selfcompassion and symptoms among individuals with BD. Study results have important implications for how we understand mechanisms underpinning symptoms in BD.

\subsection{Bivariate associations}

As hypothesised, maladaptive perfectionism was positively associated with self-reported and clinicianrated depression, anxiety and emotion regulation difficulties. Results are consistent with previous studies that found higher levels of perfectionism to be associated with symptoms of depression and anxiety among individuals with BD (Hewitt et al., 1998; Corry et al., 2013; Corry et al., 2017). We did not find an association between maladaptive perfectionism and manic symptoms however, running contrary to other studies (Corry et al., 2013; Alloy et al., 2009; Lam et al., 2004). Inconsistencies may be due in part to the use of different measures across studies. In the present study, a clinician- -rated measure of manic symptoms (the YMRS) was used rather than self-report measures used in other studies (e.g., the General Behaviour Inventory). Further, certain dimensions of perfectionism may be more relevant for specific mood states. For example, among those with $\mathrm{BD}$, associations between socially-prescribed perfectionism (i.e. the individual perceives that others have high standards for them) and manic symptoms have been reported; while self-oriented perfectionism (i.e. the individual has high standards for themselves) has been associated with depressive symptoms (Corry et al., 2017; Hewitt et al., 1998). Ongoing investigation of associations between differing dimensions of perfectionism and mood states in $\mathrm{BD}$ are required.

Turning to self-compassion, associations were in predicted directions, with lower levels of selfcompassion correlating with greater self-reported and clinician-rated depression, anxiety and emotion 
regulation difficulties. Study results align with a previous meta-analysis demonstrating negative relationships between self-compassion and symptoms of depression and anxiety in clinical and nonclinical populations (MacBeth and Gumley, 2012). Lower self-compassion has been reported among those with MDD relative to non-clinical controls, even when controlling for depressive symptoms (Krieger et al., 2013). Significant inverse correlations between self-compassion and emotion regulation difficulties have been reported in other studies (Roemer and Orsillo, 2010; Vettese et al., 2011; Krieger et al., 2013). Our findings indicate that the protective effects of self-compassion observed across mental health conditions applies in BD. Notably in the present study, self-compassion was not associated with manic symptoms. These results are broadly consistent with an earlier study of selfcompassion in a BD sample (Døssing et al., 2015), in which self-compassion was not significantly related to the annual frequency of past manic and hypomanic episodes. Self-compassion represents a kind and nurturing attitude specifically towards adversity or negative states (Neff, 2003). Mania is often (but not always) regarded as a positive state, with euphoric mood and inflated self-esteem, and thus self-compassion scales may not capture attitudes towards these manic symptoms well. Low levels of self-compassion may therefore have particular relevance for depressive and anxious symptoms in BD.

\subsection{Self-compassion as a mediator between maladaptive perfectionism and BD symptoms}

Our hypothesis that self-compassion would mediate the relationship between maladaptive perfectionism and BD symptoms was partially supported. Self-compassion was found to partially mediate the impact of maladaptive perfectionism on self-reported depression (but not clinician-rated depression), anxiety and emotion regulation difficulties. Inconsistencies between clinician-rated and self-reported outcomes of depression are common in the literature, suggesting such measures are not interchangeable despite moderate correlations (e.g. Uher et al., 2012; Cuijpers et al., 2010). In the present study, lack of mediation for the former, but not the latter, may be due to differing scale content and relative weightings of different symptom dimensions. Alternatively, methodological issues may contribute to discrepancies: for example, self-report measures are stable (e.g. questionnaire items remain the same across patients), whereas additional error variance is added in clinician-rated measures (despite training and inter-rater reliability) (Cuijpers et al., 2010). Despite this inconsistency, study results suggest that self-compassion represents one mechanism through which maladaptive perfectionism influences self-reported symptoms of depression, anxiety and emotion regulation difficulties in $\mathrm{BD}$.

Emotion regulation can be conceptualised as a broad set of cognitive, behavioural, and physiological processes that influence the way an individual experiences and responds to emotions (Finlay-Jones, 
2017). Emotion regulation difficulties contribute to the onset and maintenance of mood and anxiety disorders (Aldao et al., 2010). In BD, these have been characterised (as measured via the DERS) by difficulties engaging in goal-directed behaviour, impulsivity and limited access to useful strategies (Becerra et al., 2013; Van Rheenen et al., 2015). The latter two strategies were found to predict manic and depressive propensity, respectively, and increased symptom severity was associated with greater difficulties in emotion regulation in individuals with BD (Van Rheenen et al., 2015). Our results were consistent with this finding, insomuch as the DERS total score positively correlated with depression and anxiety symptoms. Self-compassion partially mediated the relationship between maladaptive perfectionism and emotion regulation difficulties. Notably, a large proportion of variance $(56 \%)$ in emotion regulation difficulties was accounted for by self-compassion and maladaptive perfectionism. These results provide support for self-compassion as a potentially key intervention target to facilitate adaptive emotion regulation.

Our findings fit with a broader theoretical and empirical literature on the centrality of self-compassion. Self-compassion has been conceptualised as a critical component in the capacity to regulate emotions (Gilbert, 2005; Gilbert, 2009). As overviewed by others (Finlay-Jones, 2017), developmental perspectives highlight the protective role of secure attachment relationships in the development of self-compassion and emotion regulation skills. Self-compassion was found to mediate the relationship between childhood maltreatment and later emotion regulation difficulties in one study (Vettese et al., 2011), while in another study, emotion regulation difficulties (as measured by the DERS) were associated with dysfunctional parenting experiences in those with BD II and borderline personality disorder (Fletcher et al., 2014). Early cultivation of self-compassion may act as a preventative process against the sequelae of $\mathrm{BD}$.

\subsection{Clinical implications}

Given evidence that self-compassion may be modifiable through psychotherapy, the present findings suggest that individuals with BD who exhibit maladaptive perfectionistic tendencies may potentially benefit from psychological interventions designed to foster self-compassion. Assisting these clients to adopt the alternative approach of extending kindness to themselves when they fall short of their own standards may buffer against depression, anxiety and emotion regulation difficulties.

Preliminary evidence from mindfulness-based cognitive therapy with self-compassion components suggests it is efficacious in assisting individuals with $B D$ to increase their capacity to regulate their emotions (Deckersbach et al., 2012). Participants in the present study are part of an ongoing international RCT (Fletcher et al., 2018) contrasting a mindfulness-based intervention that contains self-compassion elements with an active control. Once complete, this RCT will provide definitive data 
on the potential benefits of such an intervention for those with BD - and in particular, those presenting with high levels of maladaptive perfectionism.

Importantly, not all types of perfectionism are maladaptive. Striving to attain high personal standards can be adaptive and have positive consequences (Stoeber and Otto, 2006). As noted by others (Egan et al., 2011), the aim of treatment is not to remove striving for personal standards; rather, the focus is on curtailing self-evaluation based on meeting personal standards and criticism when the standards are not met. Self-compassion may allow individuals with BD to engage in goal-directed behaviour in a healthy and sustainable manner. Rather than excessively pursuing goals despite consequences (e.g., burn-out, leading to further emotional dysregulation), this approach may encourage flexibility in goal pursuit while simultaneously fostering resilience when goals are unable to be attained.

Maladaptive perfectionism is a common underlying feature across multiple psychopathologies: treatments targeting this issue may therefore reduce symptoms across a number of domains with specific benefit for those with comorbid conditions (Bieling et al., 2004). In BD, comorbidity is the norm rather than the exception, elevating transdiagnostic risk factors such as maladaptive perfectionism as an important treatment target. Our results provide a theoretical framework for selfcompassion as a potentially protective process to buffer the impact of maladaptive perfectionism on depression as well as the comorbid anxiety symptoms and emotion regulation difficulties characterising BD.

\subsection{Limitations}

The present cross-sectional study was limited to investigating statistical mediation, conclusions about causal mechanisms await longitudinal investigation (Kazdin, 2007). For example, it is plausible that BD symptoms (depression and anxiety in particular) precede maladaptive perfectionism. Findings require replication in other BD samples: our sample forms part of a larger randomised controlled trial targeting those with 'late stage' BD who were not currently within a mood episode; it is therefore unclear whether findings would extend to those earlier on in terms of stage of illness or with higher levels of current symptomatology. Indeed, examination of maladaptive perfectionism levels in BD individuals across different mood states would assist in unpacking the influence of current mood state on selfreported perfectionism. The role of maladaptive perfectionism and self-compassion in elevated mood states remains unclear. The range of YMRS scores in the present study was restricted due to few participants presenting with significant manic symptoms, therefore the reduced variability may have contributed to non-significant associations with key variables. Other sample characteristics limiting generalisability of findings include over-representation of females and individuals with a high level of education (over $60 \%$ with a university degree); the influence of these features remains unclear, with 
other studies reporting inconsistent results with respect to gender and educational differences on various aspects of perfectionism (Macsinga and Dobrita, 2010). Finally, we only examined one mediator; multiple mediation models including mindfulness, rumination and other associated variables would allow determination of their relative effects on the relationship between maladaptive perfectionism and BD symptoms. To provide experimental evidence regarding the centrality of selfcompassion, well-controlled treatment studies targeting self-compassion would be helpful.

\section{Conclusion}

Elevated levels of maladaptive perfectionism are associated with core symptoms of $B D$, and therefore may be a useful target in psychological interventions for those facing the condition. A focus on practicing self-compassion may protect against symptoms of depression and anxiety while fostering improved emotion regulation and quality of life. Self-compassion is a promising approach with a growing evidence base to support its efficacy. Further research is needed to highlight the important of self-compassion in enhancing wellbeing in those with $\mathrm{BD}$, and explore both the psychological and biological mechanisms that contribute to measurable positive change. 


\section{References}

Aldao, A., Nolen-Hoeksema, S., Schweizer, S., 2010. Emotion-regulation strategies across psychopathology: A meta-analytic review. Clin. Psychol. Rev. 30, 217-237.

Alloy, L.B., Abramson, L.Y., Walshaw, P.D., Gerstein, R.K., Keyser, J.D., Whitehouse, W.G., Urosevic, S., Nusslock, R., Hogan, M.E., Harmon-Jones, E., 2009. Behavioral approach system (BAS)relevant cognitive styles and bipolar spectrum disorders: Concurrent and prospective associations. J. Abnorm. Psychol. 118, 459-471.

Antony, M.M., Bieling, P.J., Cox, B.J., Enns, M.W., Swinson, R.P., 1998. Psychometric properties of the 42-item and 21-item versions of the Depression Anxiety Stress Scales in clinical groups and a community sample. Psychol. Assess. 10, 176-181.

Baron, R.M., Kenny, D.A., 1986. The moderator-mediator variable distinction in social psychological research: Conceptual, strategic, and statistical considerations. J. Pers. Soc. Psychol., 51, 1173-1182.

Becerra, R., Cruise, K., Murray, G., Bassett, D., Harms, C., 2013. Emotion regulation in bipolar disorder: Are emotion regulation abilities less compromised in euthymic bipolar disorder than unipolar depressive or anxiety disorders? Open J Psychiatr. 3, 1-7.

Bieling, P.J., Summerfeldt, L.J., Israeli, A.L., Antony, M.M., 2004. Perfectionism as an explanatory construct in comorbidity of axis I disorders. J. Psychopathol. Behav. Assess. 26, 193-201.

Bjureberg, J., Ljótsson, B., Tull, M.T., Hedman, E., Sahlin, H., Lundh, L.G., Bjarehed, J., DiLillo, D., Messman-Moore, T., Gumpert, C.H., Grazt, K.L., 2016. Development and Validation of a Brief Version of the Difficulties in Emotion Regulation Scale: The DERS-16. J. Psychopathol. Beh. Assess. 38, 284-296.

Braehler, C., Gumley, A., Harper, J., Wallace, S., Norrie, J., Gilbert, P., 2013. Exploring change processes in compassion focused therapy in psychosis: Results of a feasibility randomized controlled trial. Br. J. Clin. Psychol. 52, 199-214.

Corry, J., Green, M., Roberts, G., Fullerton, J.M., Schofield, P.R., Mitchell, P.B., 2017. Does perfectionism in bipolar disorder pedigrees mediate associations between anxiety/stress and mood symptoms? Int. J. Bipolar. Disord. 5, 34.

Corry, J., Green, M., Roberts, G., Frankland, A., Wright, A., Lau, P., Loo, C., Breakspear, M., Mitchell, P.B., 2013. Anxiety, stress and perfectionism in bipolar disorder. J. Affect. Disord. 151, 10161024.

Cuipers, P., Li, J., Hofmann, S.G., Andersson, G. (2010). Self-reported versus clinician-rated symptoms of depression as outcome measures in psychotherapy research on depression: a metaanalysis. Clin Psychol. Rev. 30, 768-778.

Deckersbach, T., Hölzel, B.K., Eisner, L.R., Stange, J.P., Peckham, A.D., Dougherty, D.D., Rauch, S.L., Lazar, S., Nierenberg, A.A., 2012. Mindfulness-based cognitive therapy for nonremitted patients with bipolar disorder. CNS Neurosci. Ther. 18, 133-141. 
Døssing, M., Nilsson, K.K., Svejstrup, S.R., Sørensen, V.V., Straarup, K.N., Hansen, T.B., 2015. Low selfcompassion in patients with bipolar disorder. Compr. Psychiatr. 60, 53-58.

Egan, S.J., Wade, T.D., Shafran, R., 2011. Perfectionism as a transdiagnostic process: A clinical review. Clin. Psychol. Rev. 31, 203-212.

Ferrari M, Yap K, Scott N, Einstein DA, Ciarrochi J. Self-compassion moderates the perfectionism and depression link in both adolescence and adulthood. PloS one. 2018;13(2):e0192022.

Finlay-Jones, A.L.., 2017. The relevance of self-compassion as an intervention target in mood and anxiety disorders: A narrative review based on an emotion regulation framework. Clin Psychol. 21, 90-103.

Fletcher, K., Fiona, F., Thomas, N., Michalak, E., Berk, L., Berk, M., Bowe, S., Cotton, S., Engel, L., Johnson, S.L., Jones S., Kyrios, M., Lapsley, S., Mihalopoulos, C., Perich, T., Murray, G., 2018. Web-based Intervention to Improve Quality of Life in Late Stage Bipolar Disorder (ORBIT): Randomised Controlled Trial Protocol. BMC Psychiatr., 18, 221.

Fletcher, K., Parker, G., Bayes, A., Paterson, A., McClure, G., 2014. Emotion regulation strategies in bipolar II disorder and borderline personality disorder: differences and relationships with perceived parental style. J. Affect. Disord. 157, 52-59.

Fletcher, K., Parker, G., Manicavasagar, V., 2013. Cognitive style in bipolar disorder sub-types. Psychiatr. Res. 206, 232-239.

Francis-Raniere, E.L., Alloy, L.B., Abramson, L.Y., 2006. Depressive personality styles and bipolar spectrum disorders: Prospective tests of the event congruency hypothesis. Bipolar Disord. 8, 382-399.

Gilbert, P., 2009. Introducing compassion-focused therapy. Adv. Psychiatr. Treat. 15, 199-208.

Gilbert, P., 2005. Compassion: Conceptualisations, Research and Use in Psychotherapy. Hove, Routledge, New York.

Glover, D.S., Brown, G.P., Fairburn, C.G., Shafran, R., 2007. A preliminary evaluation of cognitivebehaviour therapy for clinical perfectionism: A case series. Br. J. Clin. Psychol. 46, 85-94.

Gratz, K.L., Roemer, L., 2004. Multidimensional assessment of emotion regulation and dysregulation: Development, factor structure, and initial validation of the difficulties in emotion regulation scale. J. Psychopathol. Beh. Assess. 26, 41-54.

Hallion, L.S., Steinman, S.A., Tolin, D.F., Diefenbach, G.J., 2018. Psychometric properties of the Difficulties in Emotion Regulation Scale (DERS) and its short forms in adults With emotional disorders. Front. Psychol. 9, 539.

Hayes, A.F., Rockwood, N.J., 2017. Regression-based statistical mediation and moderation analysis in clinical research: Observations, recommendations, and implementation. Behav. Res. Ther. 98, 39-57.

Hayes, A., 2013. Introduction to mediation, moderation, and conditional process analysis: a regression-based approach. Guilford, New York. 
Hermens, M.L.M., Ader, H.J., van Hout, H.P.J., Terluin, B., van Dyck, R., de Haan, M., 2006. Administering the MADRS by telephone or face-to-face: a validity study. Ann. Gen. Psychiatry 5, 3.

Hewitt PL, Flett GL, Sherry SB, Caelian C., 2006. Trait Perfectionism Dimensions and Suicidal Behavior. In: Ellis T, ed. Cognition and suicide: Theory, research, and therapy. Washington, DC, US: American Psychological Association, pp215-235.

Hewitt, P.L., Flett, G.L., Ediger, E., Norton, G.R., Flynn, C.A., 1998. Perfectionism in chronic and state symptoms of depression. Can. J. Behav. Sci./Revue canadienne des sciences du comportement. 30, 234.

Johnson, S.L., 2015. Mania and dysregulation in goal pursuit: A review. Clin. Psychol. Rev. 25, 241262.

Johnson, S.L., Fulford, D., Carver, C.S., 2012. The double-edged sword of goal engagement: consequences of goal pursuit in bipolar disorder. Clin. Psychol. Psychother. 19, 352-362.

Jones, L., Scott, J., Haque, S., Gordon-Smith, K., Heron, J., Caesar, S., Cooper, C., Forty, L., Hyde, S., Lyon, L., Greening, J., Sham, P., Farmer, A., McGuffin, P., Jones, I., Craddock, N., 2005. Cognitive style in bipolar disorder. Br. J. Psychiatry. 187, 431-437.

Kazdin, A.E., 2007. Mediators and mechanisms of change in psychotherapy research. Annu. Rev. Clin. Psychol. 3, 1-27.

Kobak, K.A., Williams, J.B., Jeglic, E., Salvucci, D., Sharp, I.R., 2008. Face-to-face versus remote administration of the Montgomery-Asberg Depression Rating Scale using videoconference and telephone. Depress. Anx. 25, 913-919.

Koszycki, D., Thake, J., Mavounza, C., Daoust, J-P., Taljaard, M., Bradwejn, J., 2016. Preliminary investigation of a mindfulness-based intervention for social anxiety disorder that integrates compassion meditation and mindful exposure. J. Altern. Complement. Med. 22, 363-374.

Krieger, T., Altenstein, D., Baettig, I., Doerig, N., Holtforth, M.G., 2013. Self-compassion in depression: Associations with depressive symptoms, rumination, and avoidance in depressed outpatients. Beh. Ther. 44, 501-513.

Lam, D., Wright, K., Smith, N., 2004. Dysfunctional assumptions in bipolar disorder. J. Affect. Disord. 79, 193-199.

Leaviss, J., Uttley, L., 2015. Psychotherapeutic benefits of compassion-focused therapy: An early systematic review. Psychol. Med. 45, 927-945.

Lo, A., Abbott, M.J., 2013. Review of the theoretical, empirical, and clinical status of adaptive and maladaptive perfectionism. Beh. Change, 30, 96-116.

Lovibond, S.H., Lovibond, P.F., 1995. Manual for the Depression Anxiety Stress Scales. 2nd ed. Psychology Foundation, Sydney. 
Lowens, I., 2010. Compassion focused therapy for people with bipolar disorder. Int. J. Cog. Ther. 3, 172-185.

MacBeth, A., Gumley, A., 2012. Exploring compassion: A meta-analysis of the association between self-compassion and psychopathology. Clin. Psychol. Rev., 32, 545-552.

Macsinga, I., Dobrita, O., 2010. More educated, less irrational: Gender and educational differences in perfectionism and irrationality. Rom. J. Appl. Psychol., 12, 79-85.

Mansell, W., Morrison, A.P., Reid, G., Lowens, I., Tai, S., 2007. The interpretation of, and responses to, changes in internal states: an integrative cognitive model of mood swings and bipolar disorders. Behav. Cogn. Psychother. 35, 515-539.

Mehr K.E., Adams, A.C., 2016. Self-compassion as a mediator of maladaptive perfectionism and depressive symptoms in college students. J. College Stud. Psychother. 30, 132-145.

Miller, C.J., Johnson, S.L., Eisner, L., 2009. Assessment tools for adult bipolar disorder. Clin. Psychol: Sci. Prac. 16, 188-201.

Montgomery, S.A., Asberg, M., 1979. A new depression scale designed to be sensitive to change. $\mathrm{Br}$. J. Psychiatry., 134, 382-389.

Neff, K.D., Whittaker, T.A., Karl, A., 2017. Examining the factor structure of the self-compassion scale in four distinct populations: Is the use of a total scale score justified? J. Pers. Assess. 99, 596607.

Neff, K.D., 2003. The development and validation of a scale to measure self-compassion. Self and identity. 2, 223-250.

O'Garro-Moore, J.K., Adams, A.M., Abramson, L.Y., Alloy, L.B., 2015. Anxiety comorbidity in bipolar spectrum disorders: the mediational role of perfectionism in prospective depressive symptoms. J. Affect. Disord. 174, 180-187.

Preacher, K.J., Hayes, A.F., 2008. Asymptotic and resampling strategies for assessing and comparing indirect effects in multiple mediator models. Beh. Res. Meth. 40, 879-891.

Rice, K.G., Richardson, C.M., Tueller, S., 2014. The short form of the revised almost perfect scale. J. Pers. Assess. 96, 368-379.

Rice, K.G., Ashby, J.S., Slaney, R.B., 1998. Self-esteem as a mediator between perfectionism and depression: A structural equations analysis. J. Counsel. Psychol. 45, 304.

Riley, C., Lee, M., Cooper, Z., Fairburn, C.G., Shafran, R., 2007. A randomised controlled trial of cognitive-behaviour therapy for clinical perfectionism: A preliminary study. Behav. Res Ther. $45,2221-2231$.

Roemer, L., Orsillo, S.M., 2010. Mindfulness-and acceptance-based behavioral therapies in practice. Guilford Press; New York. 
Rush, A.J., Trivedi, M.H., Ibrahim, H.M., Carmody, T.J., Arnow, B., Klein, D.N., Markowitz, J.C., Ninan, P.T., Kornstein, S., Manber, R., Thase, M.E., Kocsis, J.H., Keller, M.B., 2003. The 16-Item Quick Inventory of Depressive Symptomatology (QIDS), clinician rating (QIDS-C), and self-report (QIDS-SR): a psychometric evaluation in patients with chronic major depression. Biol. Psychiatr. 54, 573-583.

Scott, J., Stanton, B., Garland, A., Ferrier, I., 2000. Cognitive vulnerability in patients with bipolar disorder. Psychol. Med. 30, 467-472.

Sheehan, D.V., Lecrubier, Y., Sheehan, K.H., Amorim, P., Janavs, J., Weiller, E., Hergueta, T., Baker, R., Dunbar, G.C., 1998. The Mini-International Neuropsychiatric Interview (M.I.N.I.): the development and validation of a structured diagnostic psychiatric interview for DSM-IV and ICD-10. J. Clin. Psychiatr. 59(suppl 20), 22-33.

Slaney, R.B., Rice, K.G., Mobley, M., Trippi, J., Ashby, J.S, 2001. The revised almost perfect scale. Meas. Eval. Couns. Dev. 34, 130.

Soler, J., Feliu-Soler, A., Elices, M., Carmona, C., Martin-Blanco, A., Simon, V., Pascual, J.C., 2015. Loving-kindness and compassion practices in dialectical behavioral therapy for borderline personality disorder. Actas Españolas de Psiquiatría. 43(Suppl. 1), 74-84.

Steele, A.L., Wade, T.D., 2008. A randomised trial investigating guided self-help to reduce perfectionism and its impact on bulimia nervosa: A pilot study. Behav. Res. Ther. 46, 13161323.

Stoeber, J., Otto, K., 2006. Positive conceptions of perfectionism: Approaches, evidence, challenges. Pers. Soc. Psychol. Rev. 10, 295-319.

Uher, R., Perlis, R.h., Placentino, A., Dernovsek, M.Z., Henigsberg, N., Mors, O., Maier, W., McGuffin, P., Farmer, A., 2012. Self-report and clinician-rated measures of depression severity: can one replace the other? Depress. Anx. 29, 1043-1049.

Urošević, S., Abramson, L.Y., Harmon-Jones, E., Alloy, L.B., 2008. Dysregulation of the behavioral approach system (BAS) in bipolar spectrum disorders: review of theory and evidence. Clin. Psychol. Rev. 28, 1188-1205.

Van Rheenen, T.E., Murray, G., Rossell, S.L., 2015. Emotion regulation in bipolar disorder: profile and utility in predicting trait mania and depression propensity. Psychiatr. Res. 225, 425-432.

Vettese, L.C., yer, C.E., Li, W.L., Wekerle, C., 2011. Does self-compassion mitigate the association between childhood maltreatment and later emotion regulation difficulties? A preliminary investigation. Int. J. Ment. Health. Addict. 9, 480.

Yang, Y., Fletcher K., Whitehead, R., Murray G., 2018. Toward new therapeutic mechanisms in Bipolar Disorder: Analog investigation of self-compassion and nonattachment to self. Front. Psychol. 9, 1848.

Young, R.C., Biggs, J.T., Ziegler, V.E., Meyer, D.A., 1978. A rating scale for mania: reliability, validity and sensitivity. Br. J. Psychiatry. 133, 429-435. 
Wright, K., Lam, D., Newsom-Davis, I., 2005. Induced mood change and dysfunctional attitudes in remitted bipolar I affective disorder. J. Abnorm. Psychol., 114, 689. 